\title{
MEDIDAS NÃO FARMACOLÓGICAS PARA PREVENÇÃO DA COVID-19 ENTRE A POPULAÇÃO IDOSA BRASILEIRA E FATORES ASSOCIADOS
}

\author{
Fernanda Maria Vieira Pereira Ávila ${ }^{1}$ \\ Wynne Pereira Nogueira² \\ Fernanda Garcia Bezerra Góes ${ }^{3}$ \\ Eliã Pinheiro Botelho ${ }^{4}$ \\ Elucir Gir ${ }^{5}$ \\ Ana Cristina de Oliveira e Silva ${ }^{6}$
}

\begin{abstract}
1 Graduada em Enfermagem. Doutora em Ciências. Professora adjunta da Universidade Federal Fluminense (UFF) vinculada ao Departamento de Enfermagem de Rio das Ostras. E-mail: fernanddamaria@hotmail.com.

2 Graduada em Enfermagem. Mestre em Enfermagem. Doutoranda em Enfermagem no Programa de Pós-Graduação em Enfermagem da Universidade Federal da Paraíba (UFPB). E-mail: wynnenogueira@hotmail.com.

3 Graduada em Enfermagem. Doutora em Enfermagem. Professora adjunta da Universidade Federal Fluminense (UFF) vinculada ao Departamento de Enfermagem de Rio das Ostras. E-mail: ferbezerra@ gmail.com.

4 Graduado em Enfermagem. Doutor em Ciências Biológicas. Professor adjunto da Universidade Federal do Pará (UFPA) vinculado a Faculdade de Enfermagem. E-mail: elipinbt@gmail.com.

5 Graduada em Enfermagem. Doutora em Enfermagem. Professora titular na Escola de Enfermagem de Ribeirão Preto da Universidade de São Paulo (USP). E-mail: egir@eerp.usp.br.

6 Graduada em Enfermagem. Doutora em Ciências. Professora adjunta da Universidade Federal da Paraíba (UFPB) vinculada ao Departamento de Enfermagem Clínica. E-mail: anacris.os@gmail.com.
\end{abstract}


Objetivo: Identificar as principais medidas preventivas contra a COVID-19 referidas por idosos do Brasil e os fatores associados. Métodos: Estudo transversal, de abordagem quantitativa, realizado entre idosos com idade igual ou superior a 60 anos de todas as regiões do Brasil no período de abril a maio de 2020. Os dados foram coletados por meio das mídias sociais como Facebook, Twitter, Instagram, WhatsApp e e-mail, norteada por um questionário on-line com questões sobre medidas preventivas contra a COVID-19. Utilizou-se o teste qui-quadrado e regressão logística adotando p<0,05. Resultados: Participaram do estudo 889 idosos, sendo a maioria mulheres (73,4\%), casada (57,0\%), com pós-graduação $(56,2 \%)$ e com idade média de 65 anos. A higiene das mãos (97,1\%), o isolamento social (93,3\%), o uso de álcool em gel (91,3\%) e a utilização de máscaras $(90,1 \%)$ foram as medidas preventivas contra a COVID-19 referidas como as mais adotadas pelos idosos. A higiene das mãos apresentou associação com a escolaridade ( $p=0,008)$; A medida isolamento social esteve associada ao sexo $(p=0,004)$, escolaridade $(p=0,000)$, idade $(p=0,012)$ e ser profissional de saúde $(p=0,003)$; $\bigcirc$ uso do álcool em gel esteve associado com ser profissional de saúde $(p=0,009)$ e escolaridade $(p=0,000) ;$ E o uso de máscaras apresentou associação com sexo $(p=0,041)$ e estado civil $(p=0,044)$. Conclusão: O conhecimento e a adoção das principais medidas preventivas contra a COVID-19 pelos idosos é fundamental para a garantia de proteção desse grupo populacional com a consequente diminuição da contaminação entre os idosos.

palavras-chave

Idoso. Prevenção de doenças. Infecções por coronavírus.

A Coronavirus Disease 2019 (COVID-19), doença infecciosa aguda, causada pelo agente etiológico viral Severe Acute Respiratory Syndrome Coronavirus-2 (SARS-CoV-2), foi identificada pela primeira vez em Wuhan, na China, em dezembro de 2019. Desde então, a infecção disseminou-se rapidamente pelo mundo e, em 30 de janeiro de 2020, a Organização Mundial da Saúde (OMS) declarou a COVID-19 como uma Emergência Internacional de Saúde Pública. 
Com a notificação de mais de 110 mil casos, a OMS declarou a pandemia de COVID-19 em 11 de março de 2020 (WHO, 2020a; WILDER-SMITH; FREEDMAN, 2020).

Os sintomas clínicos da infecção são muito amplos, os quais podem variar de um simples resfriado até uma pneumonia severa evoluindo para complicações como a Síndrome Respiratória Aguda Grave (SRAG) (LAI et al., 2020a). Apesar do perfil epidemiológico dos pacientes acometidos pela COVID-19 não estar totalmente esclarecido, estudos apontam que as formas mais graves da infecção têm maior probabilidade de ocorrer em idosos e em indivíduos com presença de comorbidades (LI et al., 2020; ETARD et al., 2020). Além disso, a mortalidade também é significativamente maior entre os idosos com condições de saúde pré-existentes, como hipertensão arterial sistêmica, doenças cardiovasculares e diabetes mellitus (BOCCARDI; RUGGIERO; MECOCCI, 2020).

Em 12 de março de 2021, após um ano da declaração da pandemia da COVID-19, a doença é responsável, em todo o mundo, por 118.058.503 de casos e 2.621.046 mortes. O Brasil com 11.202.305 de casos confirmados ocupa a $3^{\text {a }}$ posição do ranking mundial (WHO, 2020b). Diante do crescente número de casos da COVID-19 pelo mundo, pela rapidez de disseminação, severidade e dificuldades para sua contenção, devido à ausência de tratamentos eficazes, $\mathrm{o}$ acesso e a disponibilidade limitada de vacinas, as medidas não farmacológicas permanecem como a principal estratégia de prevenção e controle recomendadas pela OMS (BITTENCOURT, 2020). As medidas não farmacológicas são intervenções eficientes que podem ser implementadas para retardar a propagação do vírus, incluindo: higienização das mãos, etiqueta respiratória, uso de máscaras, além do isolamento social de toda a população, especialmente, a população mais vulnerável, dentre elas, a população idosa (MACHIDA et al., 2020). Estudos realizados na China apontam que a taxa de mortalidade entre os idosos é quase quatro vezes maior que a média geral (BOCCARDI; RUGGIERO; MECOCCI, 2020; LIU et al., 2020). Desse modo, os idosos se tornaram um grupo populacional de destaque no contexto da pandemia da COVID-19 devido ao risco de gravidade pela idade avançada diante da imunossenescência aumentar a vulnerabilidade às doenças infectocontagiosas e os prognósticos desfavoráveis para aqueles com doenças crônicas (ZHANG, 2020a; NUNES et al., 2020).

A fim de garantir a proteção e segurança desse grupo populacional, a medida inicialmente recomendada para esse público foi o distanciamento social. Essa ação busca reduzir a transmissão e a propagação do agente infeccioso na população, por meio da minimização de contato físico entre indivíduos (ECDC, 2020). Embora seja considerada uma importante ferramenta de 
controle da infecção do SARS-CoV-2, confirma-se também como uma medida desafiadora, visto que pode provocar impactos negativos no setor econômico, nas relações sociais e no bem-estar psicológico da população, principalmente da população idosa (RUBIN, 2020). Tendo em vista a importância do distanciamento social para prevenção e segurança dos idosos, outras medidas não farmacológicas foram determinadas e que devem estar associadas para assegurar maior proteção contra a COVID-19 nessa população. Dentre elas, estão o uso de máscaras pela população, a etiqueta respiratória e a higienização regular das mãos. Nesse ínterim, o objetivo deste estudo foi identificar as principais medidas preventivas contra a COVID-19 referidas por idosos do Brasil e os fatores associados.

\section{Métodos}

Trata-se de um estudo transversal de abordagem quantitativa que foi realizado nas cinco regiões do Brasil (Sudeste, Norte, Nordeste, Centro Oeste e Sul) no período de abril a maio de 2020 entre a população idosa brasileira. De acordo com o último censo do Instituto Brasileiro de Geografia e Estatística (IBGE, 2010), a população de idosos no Brasil com 60 anos ou mais era de aproximadamente 20.590.626 indivíduos. A partir desse número foi realizado um cálculo amostral adotando $5 \%$ de margem de erro, 95\% de confiabilidade, prevalência estimada de $50 \%$ e poder do teste de $80 \%$, obtendo-se uma amostra mínima de aproximadamente 386 participantes. Foram incluídos idosos com idade maior ou igual a 60 anos e que respondessem de maneira completa ao questionário enviado via on-line. Idosos estrangeiros residentes no Brasil ou que estivessem no país por um período determinado não foram considerados elegíveis.

A coleta de dados foi realizada por meio de mídias sociais como Facebook, Twitter, Instagram, WhatsApp e e-mail. O link da pesquisa foi enviado pelos pesquisadores e multiplicadores da pesquisa. Ao clicar no link, o entrevistado era direcionado a plataforma do Google Forms na qual constava o Termo de Consentimento Livre e Esclarecido on-line (TCLE) na página inicial. Se o indivíduo concordasse em participar do estudo, ele era direcionado aos questionários: 1- Informações demográficas e 2- Questionário de medidas preventivas contra a COVID-19. Caso contrário, ele era redirecionado aos agradecimentos na página final. Os dados coletados foram exportados para planilha do programa Microsoft OfficeExcel ${ }^{\circledR}$ e analisados pelo software IBM®SPSS, versão 20.0. Para a caracterização sociodemográfica dos idosos, utilizou-se análise estatística 
descritiva com medidas de frequência absoluta, relativa e de dispersão (Desvio-Padrão - DP). As variáveis-desfecho (dependentes) foram higiene das mãos, uso de máscaras, isolamento social, uso de álcool em gel, descontaminação de itens e locais e cuidados com a imunidade. As variáveis independentes foram: região, sexo, faixa etária, escolaridade, renda familiar, situação conjugal e ser profissional da saúde. O teste de qui-quadrado foi utilizado para examinar as associações entre as variáveis sociodemográficas e as medidas preventivas contra a COVID-19 que apresentaram maior frequência. Após, realizou-se regressão logística binária utilizando as variáveis que apresentaram associações com valor de $p<0,20$. Foi considerado $p<0,05$.

O projeto foi encaminhado via Comitê de Ética da Universidade Federal Fluminense e aprovado pela Comissão Nacional de Ética em Pesquisa (CONEP) sob número de aprovação 3.971.512. Todos os aspectos éticos foram contemplados para sua realização segundo as Resoluções Brasileiras 466/2012 e 510/2016.

\section{Resultados}

Participaram do estudo 899 idosos, de todas as regiões do Brasil, com maior expressividade na região Sudeste com $504(56,1 \%)$ idosos. A maior parte dos participantes foi de mulheres, 660 (73,4\%), com pós-graduação 550 (56,2\%) e idade média de 65 anos $( \pm 4,7)$, sendo a mínima 60 e a máxima 94 anos. A maioria dos idosos referiu ser casada $512(57,0 \%)$ e não ser profissional de saúde $580(64,5 \%)$, conforme mostra a Tabela 1.

Tabela 1 - Caracterização dos idosos segundo variáveis demográficas e individuais. ( $n=899)$. Brasil, 2020.

\begin{tabular}{lc}
\hline Variáveis & $\mathbf{n}(\%)$ \\
\hline Regiões do país & \\
Norte & $34(3,8)$ \\
Nordeste & $209(23,2)$ \\
Centro-oeste & $79(8,8)$ \\
Sudeste & $504(56,1)$ \\
Sul & $73(8,1)$ \\
\hline
\end{tabular}




\begin{tabular}{lc}
\hline Variáveis & $\mathbf{n}(\%)$ \\
\hline Sexo & $239(26,6)$ \\
Masculino & $660(73,4)$ \\
Feminino & \\
Idade (anos) & $511(56,8)$ \\
60 a 64 & $252(28,0)$ \\
65 a 69 & $136(15,1)$ \\
70 ou mais & \\
Renda (salários-mínimos) & $218(24,2)$ \\
Até 4 & $681(75,8)$ \\
5 ou mais & \\
Escolaridade & $29(3,2)$ \\
Fundamental & $102(11,3)$ \\
Médio & $263(29,3)$ \\
Superior & $519(35,5)$ \\
Pós-graduação & $505(56,2)$ \\
Estado civil & \\
Solteiro & $130(14,5)$ \\
Casado & $512(57,0)$ \\
Separado/divorciado & $(19,0)$ \\
Não & \\
\hline Srofissional de saúde & \\
\hline
\end{tabular}

Fonte: elaboração própria a partir dos dados da investigação.

Dentre as medidas citadas para prevenir a COVID-19, a higiene das mãos obteve a maior frequência, somando 873 (97,1\%), seguida por isolamento social, 839 (93,3\%), uso de álcool em gel, 821 (91,3\%), uso de máscaras, 810 (90,1\%), dentre outras (Tabela 2). 
Tabela 2 - Medidas preventivas referidas pelos idosos contra a COVID-19. (n=899). Brasil, 2020.

\begin{tabular}{lc}
\hline Variáveis & $\mathbf{n}(\%)$ \\
\hline Lavagem das mãos & $873(97,1)$ \\
Sim & $26(2,9)$ \\
Não & \\
Isolamento social & $839(93,3)$ \\
Sim & $60(6,7)$ \\
Não & \\
Uso de álcool em gel & $821(91,3)$ \\
Sim & $78(8,7)$ \\
Não & \\
Uso de máscaras & $810(90,1)$ \\
Sim & $89(9,9)$ \\
Não & \\
Descontaminação de itens e locais & $888(98,8)$ \\
Sim & $11(1,2)$ \\
Não & $53(5,9)$ \\
Cuidados com a imunidade & \\
Não & \\
\hline Sim & \\
\hline
\end{tabular}

Fonte: elaboração própria a partir dos dados da investigação.

Na associação entre as variáveis e as quatro principais medidas referidas e utilizadas pelos idosos contra a COVID-19, a higiene das mãos apresentou associação com a escolaridade $(p=0,028)$ e renda $(p=0,029)$; a medida isolamento social esteve associada ao sexo $(p=0,015)$ e escolaridade $(p=0,041)$; o uso do álcool em gel com a região do país $(p=0,004)$, escolaridade $(p=0,000)$, renda $(p=0,001)$ e ser profissional de saúde ( $p=0,027)$; e o uso de máscaras apresentou associação com escolaridade $(p=0,001)$ e ser profissional de saúde $(p=0,025)$ (Tabela 3$)$. 


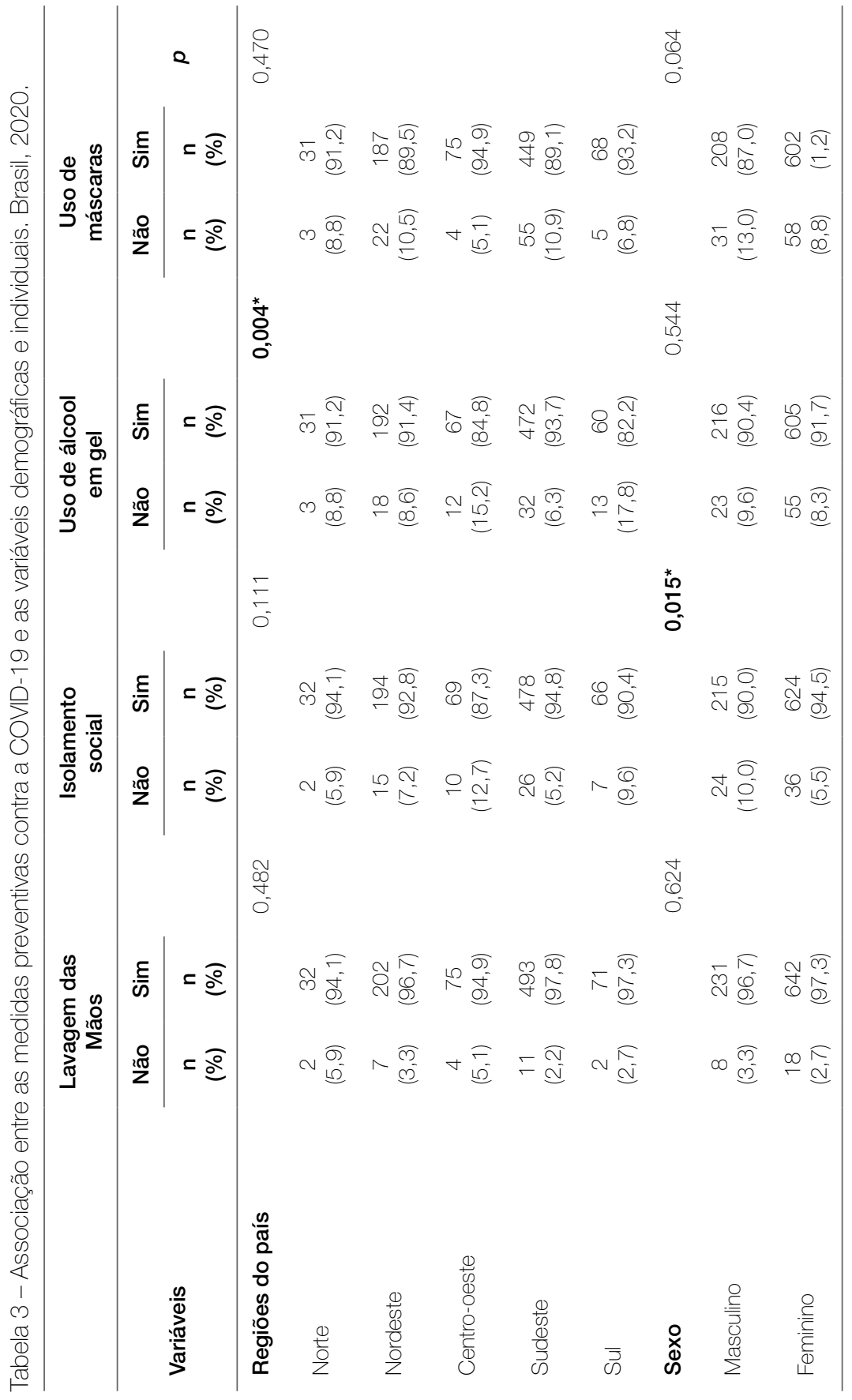




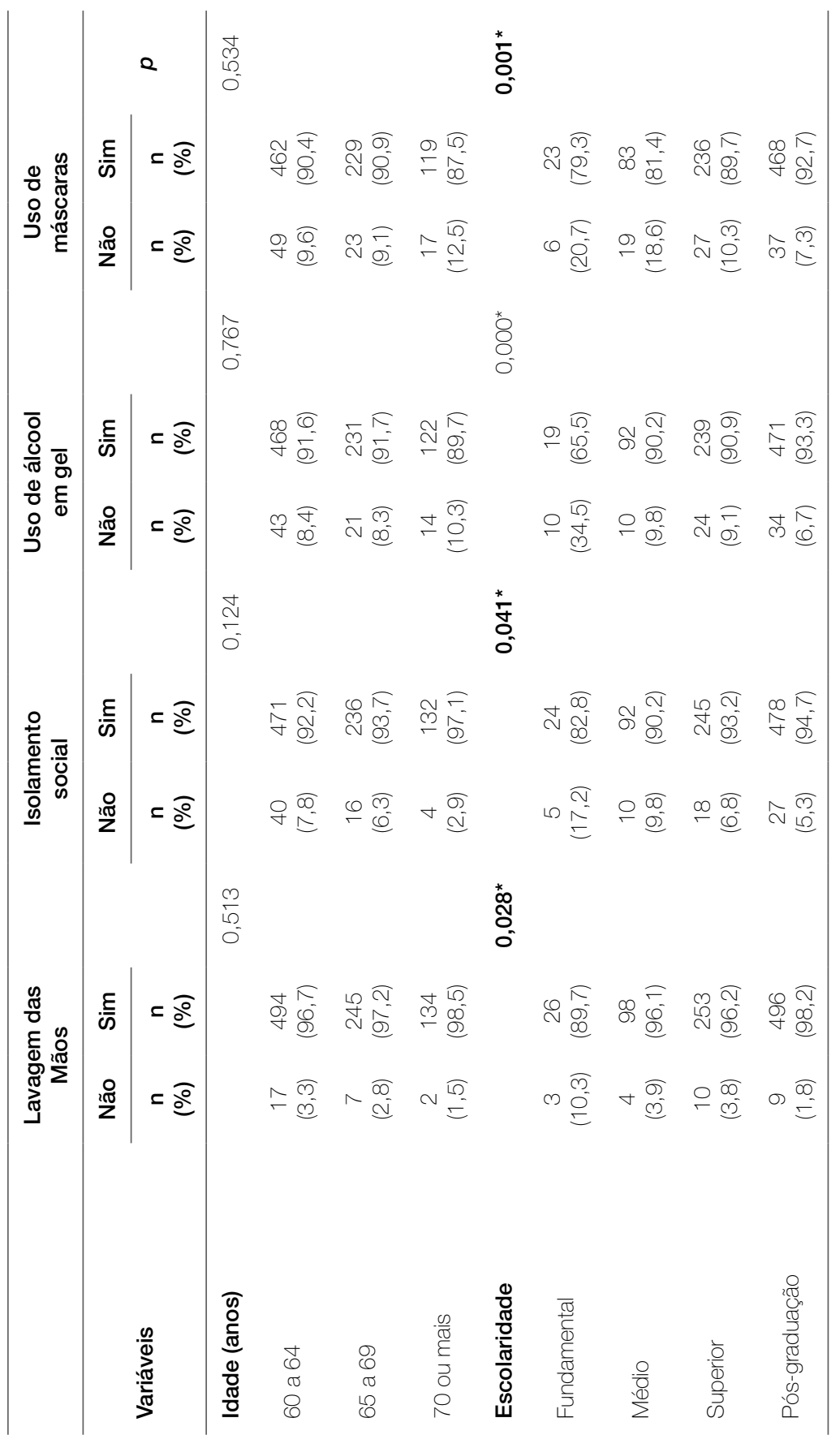

0
0
0
$\leftarrow$
$\square$
$\varangle$

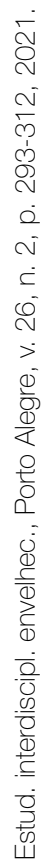




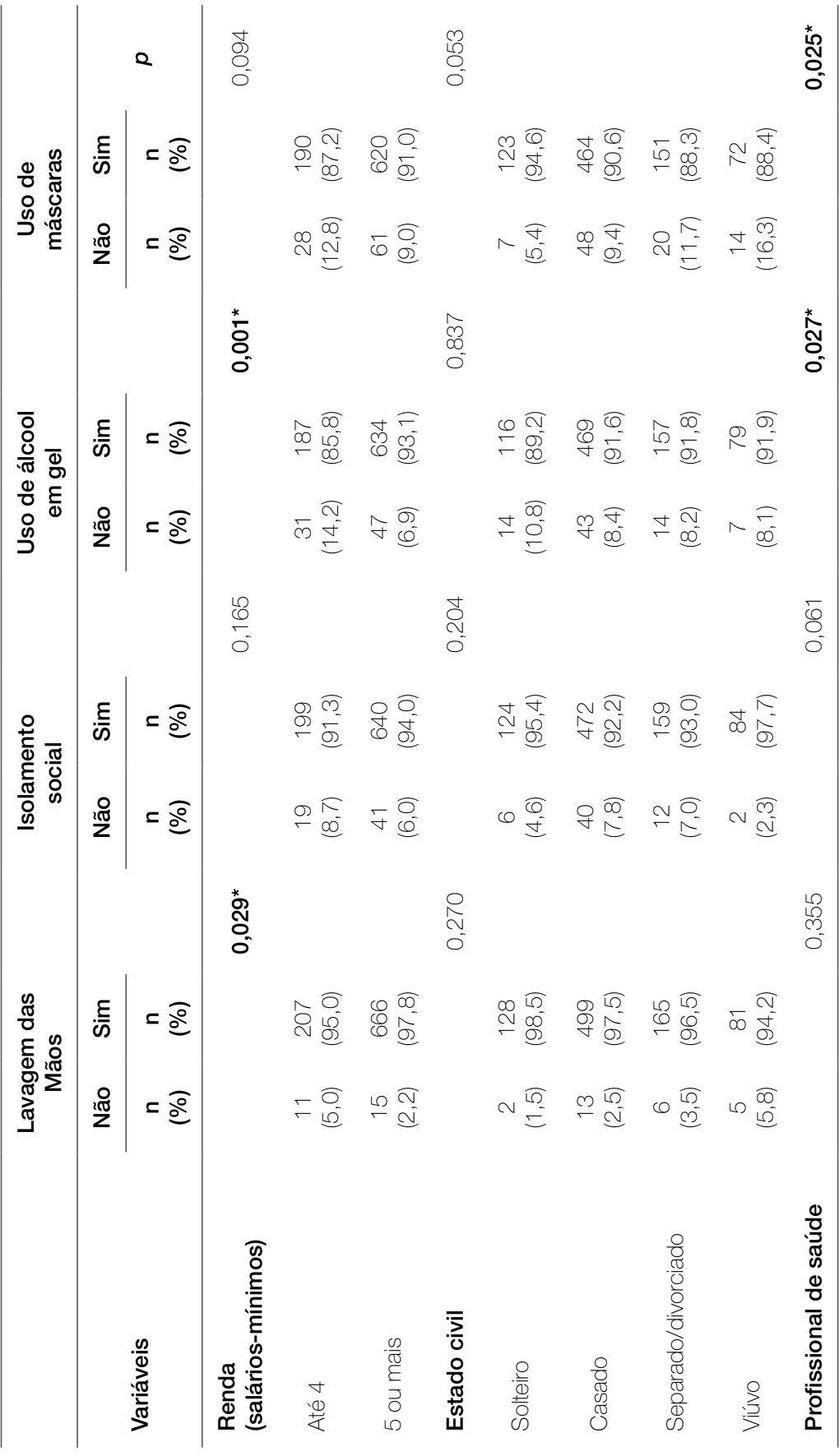




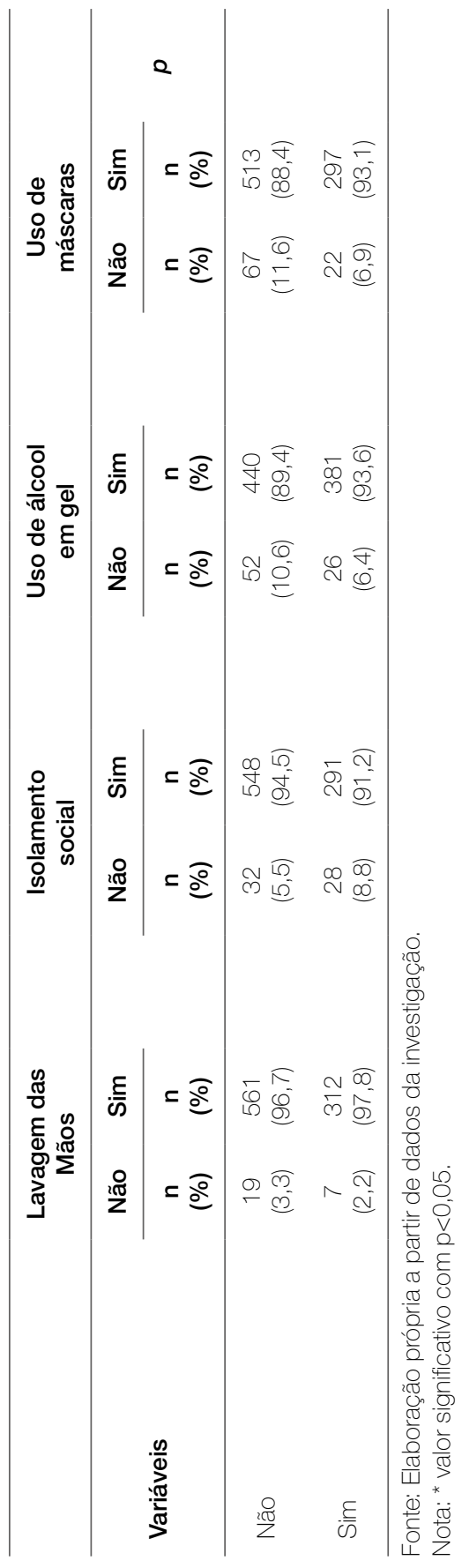


Na regressão logística, conforme mostra a Tabela 4, apenas a variável escolaridade permaneceu associada à higiene das mãos. Assim, ter pós-graduação aumentou em seis vezes as chances de realizar esta prática $(\mathrm{OR}=6,36$; $p=0,008)$. Quanto ao isolamento social, as variáveis sexo, escolaridade, idade e ser profissional de saúde se mantiveram correlacionadas a essa prática preventiva. Ser mulher aumentou em cerca de duas vezes as chances de adotar esta medida $(\mathrm{OR}=2,26 ; p=0,004)$. Ademais, ter pós-graduação $(\mathrm{OR}=8,35 ; p=0,000)$ e mais de 70 anos $(\mathrm{OR}=4,04 ; p=0,012)$ maiores são as chances para a adoção do isolamento social como medida para prevenir a COVID-19. Por outro lado, não ser profissional de saúde reduziu tais chances $(\mathrm{OR}=0,40 ; p=0,003)$.

No que se refere ao uso de álcool gel para a higienização das mãos, permaneceram associadas as variáveis escolaridade e ser profissional de saúde. Portanto, ser profissional da saúde aumentou em duas vezes as chances da realização desta prática $(\mathrm{OR}=2,20 ; p=0,009)$. $\mathrm{E}$, ainda, quanto maior o nível de escolaridade, maiores as chances de realização, com destaque para ter pós-graduação, com um aumento de aproximadamente cinco vezes $(\mathrm{OR}=4,94$; $p=0,000$ ). Com relação ao uso de máscaras, as variáveis sexo e estado civil apresentaram associação na regressão logística, pela qual se evidenciou que ser do sexo feminino aumenta em quase duas vezes as chances de uso $(\mathrm{OR}=1,70$; $p=0,041)$ e ser viúvo(a) diminui duas vezes as chances $(\mathrm{OR}=0,36 ; p=0,044)$ de adoção deste equipamento para prevenir a COVID-19. 
Tabela 4 - Razões de chances pela regressão logística binária para utilização das medidas preventivas contra a COVID-19. Brasil. 2020.

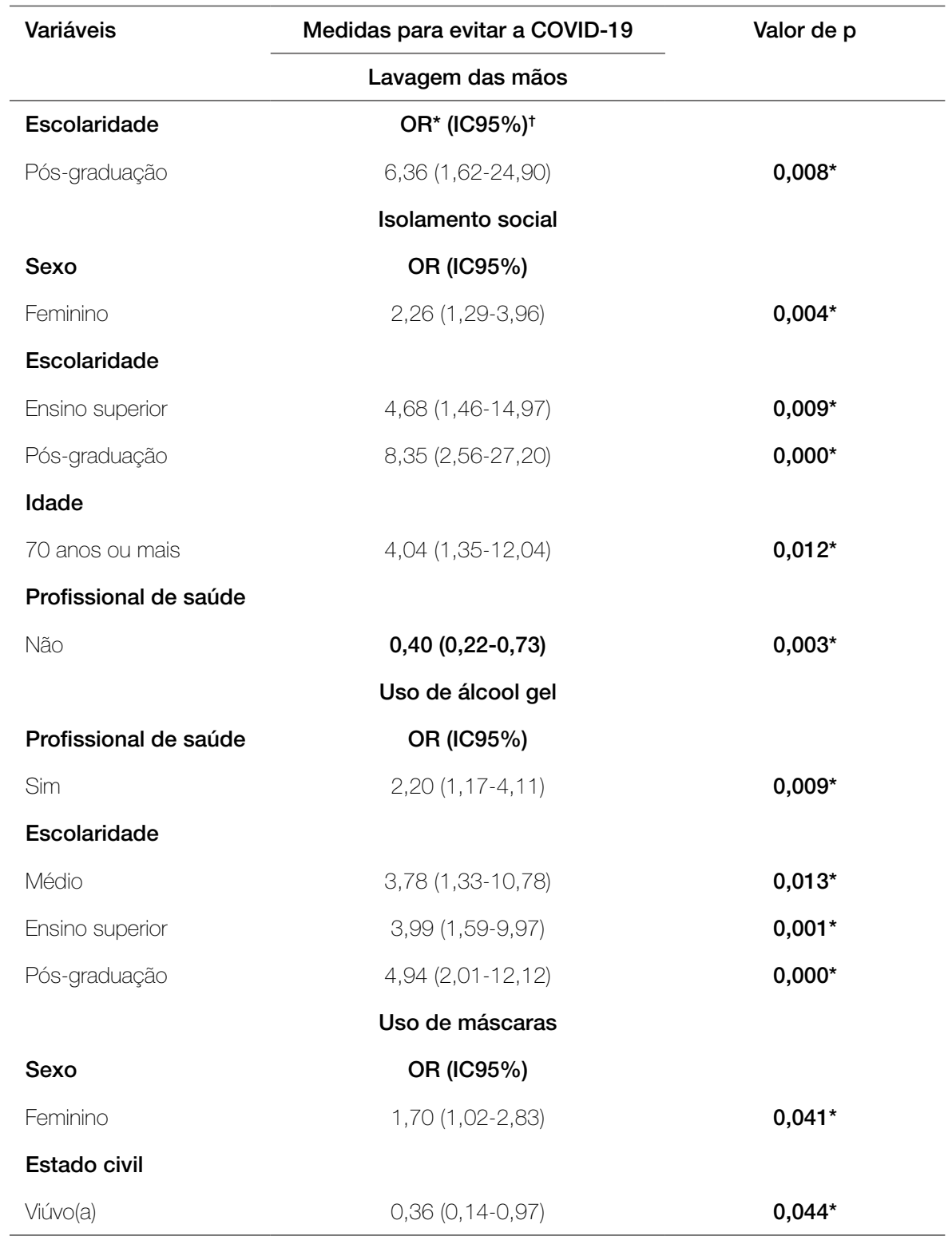

Fonte: Elaboração própria a partir de dados da investigação.

Nota: * valor significativo com p<0,05, OR: oddsratio, IC: Intervalo de Confiança. 
Os resultados do presente estudo indicam as principais medidas de prevenção relatadas pelos idosos contra a COVID-19 e as associações entre variáveis demográficas e individuais. São dados que fornecem importantes subsídios para as ações de prevenção nesta população, uma vez que permitem conhecer quais as medidas preventivas que foram mais referidas e as que necessitam de uma maior atenção para uma eventual adesão pela pessoa idosa para prevenção da COVID-19. As intervenções não farmacológicas tornaram-se componentes essenciais da resposta de saúde pública aos surtos de COVID-19. O isolamento social, o uso de máscaras e a higiene das mãos estão entre essas medidas que visam reduzir a transmissão do vírus (CDC, 2020), principalmente pelo estrato populacional vulnerável: a população idosa. Estudo realizado na Itália mostrou que os idosos por reconhecerem ser um grupo vulnerável à COVID-19, utilizavam em maior escala as medidas preventivas (higiene de mãos frequente e distanciamento social) em relação às outras faixas etárias (BARARI et al., 2020). Tal fato coaduna com os resultados do presente estudo, no qual se observou que a higiene das mãos foi uma das medidas preventivas mais relatadas pelos idosos e esteve associada com a escolaridade.

Estudos apontam que a higiene das mãos com álcool em gel ou com água e sabão reduz a propagação exponencial do vírus da COVID-19 (MA et al., 2020; LAI et al., 2020b). O nível de escolaridade é um fator importante para a adesão a essa medida, uma vez que um menor nível de escolaridade reflete em um menor acesso à informação (OLIVEIRA; THOMAZ; SILVA, 2008). É possível observar no estudo que a maioria dos idosos participantes possui pós-graduação. Neste ínterim, a alta frequência de relato da higienização das mãos como forma de prevenção pode estar diretamente relacionada com o acesso à informação sobre a importância da medida como barreira protetiva para a COVID-19.

O isolamento social também esteve entre as medidas preventivas mais relatadas pela população idosa. A intervenção visa à redução da transmissibilidade entre os indivíduos e consequente redução da curva epidêmica (ECDC, 2020). As respostas eficazes dessas medidas são baseadas em estudos observacionais e de modelagem matemática, pois estudos sobre a adesão e o impacto na redução ainda são escassos (FERGUSON et al., 2020). Esta medida foi inicialmente pensada para a população idosa por ser considerada de alto risco. No entanto, o distanciamento social impede o encontro de pessoas, seja em casa, lugares públicos e com a família, o que pode trazer repercussões negativas na saúde mental da pessoa idosa, tornando-se uma estratégia desafiadora 
(BAKER; CLARK, 2020). Embora o estudo em questão não tenha avaliado a adesão ao isolamento social, a alta frequência relatada pelos idosos como forma de prevenção da COVID-19 torna-se um importante dado. A medida esteve associada ao sexo feminino, escolaridade, idade e ser profissional de saúde.

Como mencionado anteriormente, a escolaridade é um importante fator relacionado ao acesso à informação e à prática da medida preventiva. Na Arábia Saudita o nível de conhecimento sobre a COVID-19 estava diretamente associado ao nível escolar e à renda familiar, e a prática de medidas preventivas apresentava associação com o sexo feminino (ALAHDAL; BASINGAB; ALOTAIBI, 2020). Pesquisa desenvolvida no Japão, que analisou a implementação das medidas de proteção por meio de uma avaliação autorreferida mostrou que a prevalência da prática do distanciamento social entre os homens foi baixa e maior entre os idosos, com a necessidade de estratégias de medidas educacionais voltadas a um gênero específico e aos jovens e adultos (MACHIDA et al., 2020).

Um aspecto que chama a atenção foi a associação entre ser profissional de saúde com a prática do isolamento social relatado pelos idosos. O estudo mostrou que ser profissional de saúde reduz as chances da prática do isolamento social. Os idosos, principal grupo de risco para a COVID-19, que atuam na assistência deveriam ser realocados ou dispensados do trabalho durante a pandemia. No entanto, observa-se que na prática os idosos permanecem em suas atividades, tornando-se um desafio para a prática do isolamento social. O direito à vida e a execução do trabalho em condições seguras e protegidas é uma meta a ser incorporada nas ações de enfrentamento da epidemia (HELIOTERIO et al., 2020).

Outra importante medida autorreferida pelos idosos foi o uso de álcool em gel. Essa medida deve estar diretamente interligada com a higienização das mãos, visto que seu principal uso na pandemia da COVID-19 voltou-se para este fim, além da descontaminação de superfícies e objetos, como precaução padrão contra as doenças (BRASIL, 2020). Uma característica significativa associada com o uso de álcool em gel pelos idosos foi ser profissional de saúde e possuir um maior nível de escolaridade.

Sabe-se que os profissionais de saúde foram os mais suscetíveis ao contágio pelo novo coronavírus, especialmente aqueles que lidam diretamente com pacientes diagnosticados com a infecção. Desse modo, a atenção para a adesão ao conjunto de medidas de prevenção da COVID-19 foi redobrada, especialmente entre profissionais de saúde que fazem parte do grupo de risco, como os idosos (ZHANG et al., 2020).

Em relação à alta transmissibilidade do SARS-CoV-2, as máscaras tornaram-se acessório indispensável e obrigatório para uso da população 
em geral durante a pandemia e está incluída entre as medidas preventivas relatadas pelos idosos do Brasil participantes do presente estudo. $\mathrm{O}$ uso de máscaras tem a finalidade de autoproteção contra a doença e, também, para prevenir a transmissão de patógenos entre os indivíduos doentes e saudáveis (ZHONG et al., 2020). A frequência de uso se deu gradativamente, de acordo com o avanço da pandemia nas regiões brasileiras. Observou-se que o uso de máscaras entre os idosos esteve associado com o sexo feminino e com o estado civil viúvo(a). A associação pode estar relacionada a uma maior preocupação das mulheres com a saúde em comparação com os homens, além de terem mais chances para aceitar as recomendações propostas pelos governos ou entidades (HUANG et al., 2020). Ser viúvo(a) reduziu as chances da utilização de máscaras pelos idosos. $\mathrm{O}$ apoio familiar ou a presença de um companheiro(a) pode reforçar os idosos na adoção de medidas protetivas para a sua saúde, além de influenciar no comportamento de autocuidado (SO et al., 2014). Tendo em vista a disponibilidade limitada de vacinas para a população mundial e de tratamento específicos e eficazes contra a COVID-19, as medidas não farmacológicas devem ser priorizadas no enfrentamento da pandemia, principalmente entre os grupos populacionais vulneráveis como os idosos.

Como limitações do presente estudo, destacam-se: a exclusão dos analfabetos digitais, o impedimento do auxílio ao participante quando o mesmo não compreende alguma pergunta e a impossibilidade do conhecimento das circunstâncias em que o questionário foi respondido.

\section{Conclusão}

O estudo evidenciou que a higiene das mãos, o isolamento social, o uso de álcool em gel e o uso de máscaras foram as medidas não farmacológicas de prevenção contra a COVID-19 referidas como as mais adotadas pelos idosos. $\mathrm{O}$ sexo, a escolaridade, ser profissional de saúde foram os principais fatores associados a adoção das medidas preventivas. $\mathrm{O}$ conhecimento sobre as medidas preventivas na população idosa frente à pandemia da COVID-19 irá permitir o planejamento de estratégias para a garantia de proteção desse grupo populacional, com a diminuição da contaminação entre os idosos, visto que é um estrato populacional de risco das formas mais graves da infecção. É premente ações de educação em saúde para a população idosa. Esta pesquisa mostrou a importância da escolaridade na adoção das medidas não farmacológicas de enfrentamento da COVID-19. Projetos voltados para educação em saúde, educação permanente e educação continuada devem fazer parte da agenda de intervenções em todos os níveis de atenção à saúde priorizando o momento de pandemia e pós-pandemia. 
NON-PHARMACEUTICAL MEASURES

TO PREVENT COVID-19 AMONG THE ELDERLY

BRAZILIAN POPULATION AND FACTORS ASSOCIATED

abstract

Objective: To identify the main preventive measures against COVID-19 mentioned by the elderly of Brazil and the associated factors. Methods: A cross-sectional study, with a quantitative approach, was conducted with elderly people aged 60 years or over from all regions of Brazil during April through May, 2020. The data were collected through social media such as Facebook, Twitter, Instagram, WhatsApp and email, guided by an online questionnaire with questions about preventive measures against COVID-19. Chi-square test and logistic regression were used, adopting p <0.05. Results: 889 elderly people participated in the study, the majority of were women (73.4\%), married (57.0\%), with a graduate degree (56.2\%) and with an average age of 65 years. The hand hygiene (97,1\%), the social isolation (93,3\%), use of alcohol gel $(91,3 \%)$ and the use of masks $(90,1 \%)$, were the preventive measures against COVID-19 referred to as the most adopted by the elderly. Hand hygiene was associated with education ( $p=0.008)$; The measure of social isolation was associated with gender $(p=0.004)$, education $(p=0.000)$, age $(p=0.012)$ and being a health professional $(p=$ 0.003); The use of alcohol gel was associated with being a health professional ( $p=0.009$ ) and education ( $p=0.000)$; And the use of masks was associated with sex $(p=0.041)$ and marital status ( $p=0.044)$. Conclusion: The knowledge and adoption of the main preventive measures against COVID-19 by the elderly is essential to ensure the protection of this population group with the consequent decrease in contamination among the elderly.

keywords

Aged. Disease Prevention. Coronavirus infections. 
ALAHDAL, Hadil; BASINGAB, Fatemah; ALOTAIBI, Reem. An analytical study on the awareness, attitude and practice during the COVID-19 pandemic in Ryadah, Saudi Arabia. Journal of Infection and Public Health, Saudi Arabian, v. 13, p. 1446-1452, 2020. Disponivel em: https://www.sciencedirect.com/science/article/pii/\$1876034120305256. Acesso em: 16 nov. 2020.

BAKER, Edward; CLARK, Louise. Biopsychopharmacosocial approach to assess impact of social distancing and isolation on mental health in older adults. British Journal of Community Nursing, United Kingdom, v. 25, n. 5, p. 231-238, 2020. Disponível em: https://www.magonlinelibrary.com/doi/abs/10.12968/bjcn.2020.25.5.231. Acesso em: 7 nov. 2020.

BARARI, Soubhik et al. Evaluating COVID-19 public health messaging in Italy: self-reported compliance and growing mental health concerns [ahead of print]. MedRxiv. 2020. Disponível em: https://www.medrxiv.org/content/10.1101/2020.03.27.20042 820v2. Acesso em: 2 nov. 2020

BITTENCOURT, Renato Nunes. Pandemia, isolamento social e colapso global. Revista Espaço Acadêmico, Maringá, v. 19, n. 221, p. 168-178, 2020. Disponível em: http:// periodicos.uem.br/ojs/index.php/EspacoAcademico/article/view/52827. Acesso em: 26 out. 2020.

BOCCARDI, Virgínia; RUGGIERO, Carmelinda; MECOCCl, Patrizia. COVID-19: A Geriatric Emergency. Geriatrics, Switzerland, v. 5, n. 2, 2020. Disponível em: https://www.mdpi. com/2308-3417/5/2/24. Acesso em: 5 nov. 2020.

BRASIL. Ministério da Saúde. Governo do Estado do Ceará. Secretaria de Saúde Boletim Epidemiológico. Doença pelo novo Coronavírus (COVID-19). 2020. Disponível em: https://www.saude.ce.gov.br/download/boletins/. Acesso em: 2 nov. 2020.

CENTERS FOR DISEASE CONTROL AND PREVENTION (CDC). 2019 Novel coronavirus, Wuhan, China. 2020. Disponivel em: https://www.cdc.gov/coronavirus/2019-nCoV/ summary.html. Acesso em: 28 out. 2020.

EUROPEAN CENTRE FOR DISEASE PREVENTION AND CONTROL (ECDC). Considerations relating to social distancing measures in response to COVID-19-second update. Stockholm: ECDC; 2020. Disponível em: https://www.ecdc.europa.eu/en/publications-data/considerations-relating-social-distancing-measures-response-covid-19-second. Acesso em: 27 out. 2020.

ETARD, Jean-François et al. Potential lethal outbreak of coronavirus disease (COVID19) among the elderly in retirement homes and long-term facilities, France, Mar. 2020. Eurosurveillance, European Union, v. 25, n. 15, 2020. Disponível em: https://www. eurosurveillance.org/content/10.2807/1560-7917.ES.2020.25.15.2000448. Acesso em: 5 nov. 2020

FERGUSON, Neil et al. Impact of non-pharmaceutical interventions (NPIs) to reduce COVID-19 mortality and healthcare demand. Imperial College London, London, 2020. Disponível em: https://www.imperial.ac.uk/media/imperial-college/medicine/sph/ide/ gida-fellowships/Imperial-College-COVID19-NPI-modelling-16-03-2020.pdf. Acesso em: 28 out. 2020.

HELIOTERIO, Margarete Costa et al. COVID-19: Por que a proteção de trabalhadores e trabalhadoras da saúde é prioritária no combate à pandemia? Trabalho, Educação e Saúde, Rio de Janeiro, v. 18, n. 3, 2020. Disponível em: http://www.scielo.br/scielo. php?script=sci_arttext\&pid=\$1981-77462020000300512\&lng=en\&nrm=iso. Acesso em: 24 nov. 2020. 
HUANG, Yu et al. Measures undertaken in China to avoid COVID-19 infection: internet-based, cross-sectional survey study. Journal of Medical Internet Research, Canadá, v. 22, n. 5, 2020. Disponível em: https://pubmed.ncbi.nlm.nih.gov/32396516/. Acesso em: 24 nov. 2020.

INSTITUTO BRASILEIRO DE GEOGRAFIA E ESTATÍSTICA (IBGE). População residente, por sexo e grupos de idade, segundo as Grandes Regiões e as Unidades da Federação. Censo demográfico, 2010. Disponível em: https://censo2010.ibge.gov.br/sinopse/index. php?dados=12. Acesso em: 21 out. 2020.

LAl, Chih-Cheng et al. Severe acute respiratory syndrome coronavirus 2 (SARS-CoV-2) and coronavirus disease-2019 (COVID-19): The epidemic and the challenges. International Journal of Antimicrobial Agents, United Kingdom, v. 55, n. 3, 2020a. Disponível em: https://www.sciencedirect.com/science/article/pii/S0924857920300674. Acesso em: 5 nov. 2020.

LAl, Shenggjie et al. Effect of non-pharmaceutical interventions to contain COVID-19 in China. Nature, Germany, n. 585, p. 410-413, 2020b. Disponível em: https://www. nature.com/articles/s41586-020-2293-x. Acesso em: 1 nov. 2020.

LI, Heng et al. Coronavirus disease 2019 (COVID-19): current status and future perspectives. International Journal of Antimicrobial Agents, United Kingdom, v. 55, n. 5, 2020. Disponível em: https://www.sciencedirect.com/science/article/abs/pii/ S0924857920301011. Acesso em: 5 nov. 2020.

LIU, Kai et al. Clinical features of COVID-19 in elderly patients: A comparison with young and middle-aged patients. The Journal of Infection, Britain, v. 80, n. 6, p. 14-18, 2020. Disponível em: https://pubmed.ncbi.nlm.nih.gov/32171866/. Acesso em: 5 nov. 2020.

MA, Qing-Xia et al. Potential utilities of mask-wearing and instant hand hygiene for fighting SARS-CoV-2. Journal of Medical Virology, United States of America, n. 92, p. 1567-1571, 2020. Disponível em: https://onlinelibrary.wiley.com/doi/abs/10.1002/ jmv.25805. Acesso em: 6 nov. 2020.

MACHIDA, Masaki et al. Adoption of personal protective measures by ordinary citizens during the COVID-19 outbreak in Japan. International Journal of Infectious Diseases, Denmark, v. 93, p. 149-144, 2020. Disponivel em: https://www.sciencedirect.com/ science/article/pii/S1201971220302307. Acesso em: 24 nov. 2020.

NUNES, Vilani Medeiros de Araújo et al. COVID-19 e o cuidado de idosos: recomendações para instituições de longa permanência. Natal: EDUFRN; 2020. Disponível em: https://repositorio.ufrn.br/jspui/handle/123456789/28754. Acesso em: 24 nov. 2020.

OLIVEIRA, Bruno Luciano Carneiro Alves; THOMAZ, Erika Barbara Abreu Fonseca; SILVA, Raimundo Antonio da. Associação da cor/raça aos indicadores de saúde para idosos no Brasil: um estudo baseado na Pesquisa Nacional por Amostra de Domicílios. Caderno de Saúde Pública, Rio de Janeiro, v. 30, n. 7, p. 1-15, 2008. Disponível em: https://www. scielo.br/scielo.php?pid=S0102-311X2014000701438\&script=sci_abstract\&tlng=pt. Acesso em: 1 nov. 2020.

RUBIN, Gideon James. The psychological effects of quarantining a city. The BMJ, United Kingdom, n. 368, p. 1-2, 2020. Disponível em: https://www.bmj.com/content/ bmj/368/bmj.m313.full.pdf. Acesso em: 28 out. 2020.

SO, Winnie Kwok Wei et al. The knowledge level and precautionary measures taken by older adults during the SARS outbreak in Hong Kong. International Journal of Nursing Studies, London, v. 41, n. 8, p. 901-909, 2020. Disponível em: https://www.ncbi.nlm. nih.gov/pmc/articles/PMC7130144/. Acesso em: 23 nov. 2020.

WILDER-SMITH, Annelies; FREEDMAN, David. Isolation, quarantine, social distancing and community containment: pivotal role for old-style public health measures in the novel coronavirus (2019-nCoV) outbreak. Journal of Travel Medicine, United Kingdom, v. 27, n. 2, 2020. Disponível em: https://academic.oup.com/jtm/article/27/2/taaa020/5735321. Acesso em: 5 nov. 2020. 
WORLD HEALTH ORGANIZATION (WHO). Novel coronavirus (2019-nCoV): situation report - 22. Geneva: WHO; 2020a. Disponivel em: https://www.who.int/docs/default-source/ coronaviruse/situation-reports/20200211-sitrep-22-ncov.pdf?sfvrsn=fb6d49b1_2. Acesso em: 5 nov. 2020.

WORLD HEALTH ORGANIZATION (WHO). Coronavirus disease (COVID-19) Dashboard. Geneva: WHO; 2020b. Disponível em: https://covid19.who.int/. Acesso em: 12 mar. 2021

ZHANG, Wenhong. Manual de Prevenção e Controle da COVID-19 segundo o Doutor Wenhong Zhang. São Paulo: Polo Books; 2020a. Disponivel em: https://www.dfl.com. br/especiais/wp-content/uploads/2020/04/manual_prev_covid19_17_04_2020.pdf. Acesso em: 7 nov. 2020

ZHANG, Zhirou et al. Protecting healthcare personnel from 2019-nCoV infection risks: lessons and suggestions. Frontiers of Medicine, China, v. 14, n. 2, p. 229-231, 2020 b. Disponível em: https://pubmed.ncbi.nlm.nih.gov/32212058/. Acesso em: 7 nov. 2020.

ZHONG, Bao-Liang et al. Knowledge, attitudes, and practices towards COVID-19 among Chinese residents during the rapid rise period of the COVID-19 outbreak: a quick online cross-sectional survey. International Journal of Biological Sciences, China, v. 16, n. 10, 2020. Disponível em: https://www.ncbi.nlm.nih.gov/pmc/articles/PMC7098034/. Acesso em: 7 nov. 2020

Data de Submissão: 22/12/2020

Data de Aprovação: 02/04/2021 